\title{
IMPACTOS AMBIENTAIS DA SUBSTITUIÇÃO DO GUINCHO ARRASTADOR POR SKIDDER DE ESTEIRAS
}

\author{
Luís Carlos de Freitas ${ }^{1}$, Carlos Cardoso Machado², Elias Silva ${ }^{2}$, Márcio Lopes da Silva ${ }^{2}$, \\ Ângelo Márcio Pinto Leite ${ }^{3}$, Haroldo Carlos Fernandes ${ }^{2}$ \\ ${ }^{1}$ Eng. Florestal, Dr., Depto. de Fitotecnia e Zootecnia, UESB, BA, Brasil - luiscarlos_ufv@yahoo.com.br \\ ${ }^{2}$ Eng. Florestal, Dr., Depto. de Engenharia Florestal, UFV, MG, Brasil - machado@ufv.br; \\ eshamir@ufv.br; malosil@ufv.br; haroldo@ufv.br \\ ${ }^{3}$ Eng. Florestal, Dr., Depto. de Engenharia Florestal, UFVJM, MG, Brasil - ampleite@yahoo.com.br
}

Recebido para publicação: 15/10/2009 - Aceito para publicação: 01/03/2011

\begin{abstract}
Resumo
Este trabalho teve como objetivo avaliar os impactos ambientais quando da mudança do subsistema tradicional (motosserra + guincho arrastador) para o inovado (motosserra + skidder de esteiras). Utilizou-se um método consolidado para avaliação de impacto ambiental da inovação tecnológica na agropecuária, que avalia o desempenho ambiental de uma tecnologia em relação àquela previamente estabelecida, adaptado para avaliação de impactos na colheita florestal. Os impactos foram analisados para indicadores dos meios físico (atmosfera, solo, água), biótico (flora e fauna) e antrópico (emprego, saúde, paisagismo e gestão). Matrizes foram elaboradas para cada indicador, sendo elas ponderadas em relação à escala de ocorrência e fator de importância. As matrizes foram preenchidas por técnicos florestais, que utilizaram coeficientes de alteração. O coeficiente de impacto resultou do produto do coeficiente de alteração pelos fatores de ponderação, sendo avaliado numa escala de -15 a +15 , determinando-se o índice geral de impacto na mesma escala. De acordo com os resultados, sete indicadores apresentaram coeficiente total médio de impacto positivo e dois coeficientes negativos. O índice geral de impacto mostrou-se positivo $(+1,44)$, indicando aspecto vantajoso no contexto ambiental quando da adoção do subsistema inovado.

Palavras chave: Inovação tecnológica; colheita florestal; impacto ambiental; indicadores.
\end{abstract}

\begin{abstract}
Environmental impacts in the replacing of the cable skidder by track skidder. This work aimed to evaluate environmental impacts of replacing traditional subsystem (Chainsaw + Cable Skidder) by an innovated subsystem (Chainsaw + Track Skidder). In order to do that, it used an already known method for evaluation of environmental impact of technological innovation on agriculture, which evaluates environmental performance of a new technology in relation to a previously established one. This method was adapted for evaluation of impacts on forest harvesting. The impacts had been analyzed in relation to the following indicators: physical (atmosphere, soil, water), biotic (plants and fauna), and anthropic (employment; health, landscaping and management). Matrices had been elaborated to each component, which had been weight in relation to scale of occurrence and factor of importance. The matrices were filled in by technicians, who used coefficients of alteration previously defined. The impact coefficient resulted from the product of alteration coefficient by weight factors, evaluated in scale of -15 to +15 . The overall impact index was determined by the same scale. According to results, seven components presented positive impact coefficient and two of them presented negative ones. The overall impact index presented positive result $(+1,44)$, which points to environmental advantage for the innovated subsystem adoption.

Keywords: Technological innovation; forest harvesting; environmental impact, indicators.
\end{abstract}

\section{INTRODUÇÃO}

A colheita florestal no Brasil passou por profundas mudanças nas últimas décadas, com grandes avanços técnicos, operacionais, ergonômicos e, sobretudo, de produtividade. Até a década de 40 do século $\mathrm{XX}$, praticamente não havia emprego de máquinas nas operações de colheita florestal, com as operações

FLORESTA, Curitiba, PR, v. 41, n. 3, p. 519-532, jul./set. 2011.

Freitas, L. C. de et al. 
de corte realizadas de forma rudimentar, com uso de machado, foices e serras. A extração também se baseava em métodos rudimentares, utilizando trabalho braçal e animal. Da década de 40 até os dias atuais, muitas mudanças ocorreram no setor, com as operações evoluindo de processo manual para semimecanizado, adquirindo um nível mais significativo em termos de mecanização. Na década de 80, as principais inovações foram a substituição das motosserras por feller-bunchers e harvesters, surgindo também os skidders, os quais passaram a substituir os tratores agrícolas adaptados para o processo de extração florestal. Na década de 90, houve uma grande modernização do processo de mecanização da colheita florestal, devido, sobretudo, à abertura das importações (MACHADO et al., 2008; MALINOVSKI et al., 2008).

Esse processo de inovação tecnológica de máquinas na colheita florestal trouxe, no entanto, um novo cenário com relação aos aspectos ambientais nessa atividade. As empresas que aderiram ao progresso tecnológico na colheita passaram a utilizar maquinários pesados nas operações, agravando assim os processos de compactação (SEIXAS; OLIVEIRA JÚNIOR, 2001) e erosão do solo (FREITAS et al., 2007). Os impactos relacionados à vegetação de sub-bosque e às brotações também foram agravados em algumas circunstâncias, principalmente pelo processo de arraste com skidders (LIRA FILHO, 1993; FREITAS, 2004). No contexto social houve muitas mudanças, como, por exemplo, a redução da mão de obra de baixa qualificação e também dos índices de acidentes (EQUIPE TÉCNICA DURATEX, 1999), a melhoria da qualificação profissional e o aumento da remuneração (FREITAS, 2008).

A inovação tecnológica da colheita florestal proporcionou grandes alterações ambientais nos aspectos físicos, bióticos e antrópicos, evidenciando assim uma atenção especial no que tange ao estudo dessa problemática decorrente do processo de modernização. Nesse contexto, é de suma importância a adoção de uma metodologia que possa quantificar tais alterações e transformá-las em coeficientes de impacto, o que possibilitará a comparação dos danos ambientais bem como o direcionamento de medidas de controle ambiental (mitigadoras ou potencializadoras) para aqueles impactos mais expressivos (FREITAS, 2008). Este trabalho teve como principal objetivo avaliar os impactos ambientais decorrentes do processo de inovação tecnológica na colheita florestal, mais especificamente na substituição de um guincho arrastador por um skidder de esteiras na extração de madeira em terreno acidentado, adotando um método adaptado para avaliação de impactos na agricultura. Os objetivos específicos foram:

- determinar, por meio de matriz de ponderação, os coeficientes de impacto ambiental para os indicadores dos aspectos físico, biótico e antrópico, quando da mudança do subsistema motosserra + guincho arrastador (tradicional) para motosserra + skidder de esteiras (inovado);

- determinar o índice geral de impacto ambiental para o processo de inovação tecnológica avaliado;

- discutir os principais fatores responsáveis pelas alterações ambientais (positivas e negativas), quando da adoção do processo de inovação tecnológica em estudo.

Uma grande vantagem do método em questão consiste no fato de permitir uma avaliação rápida dos parâmetros ambientais; já sua principal desvantagem está relacionada com o grau de subjetividade (FREITAS, 2008).

\section{MATERIAL E MÉTODOS}

\section{Caracterização das áreas de estudo}

$\mathrm{O}$ trabalho foi conduzido em povoamentos florestais pertencentes a uma empresa produtora de celulose no estado de Minas Gerais, localizada na região de Nova Era/Cocais (1956'45', de latitude sul e 4329'14', de longitude oeste), caracterizada por um relevo forte ondulado a montanhoso, com solo pouco profundo e de textura argilo-arenosa, apresentando baixa fertilidade e as seguintes condições climáticas: precipitação de $1.280 \mathrm{~mm} / \mathrm{ano}$, UR $(66 \%)$ e temperatura média de $21,2{ }^{\circ} \mathrm{C}$.

\section{Avaliação de impactos ambientais}

Existe à disposição dos avaliadores de impacto ambiental um vasto arsenal metodológico, com mais de cem métodos descritos para os mais variados propósitos e situações (BISSET, 1987; CANTER, 1996). No caso em estudo, trabalhou-se com um método consolidado para avaliação do impacto ambiental da inovação tecnológica na agropecuária (AMBITEC-AGRO), desenvolvido pela Embrapa Meio Ambiente, que avalia o desempenho ambiental de uma dada tecnologia ou metodologia em relação àquela previamente estabelecida (RODRIGUES et al., 2003). O sistema AMBITEC foi direcionado para uma avaliação dos 
impactos ambientais oriundos dos avanços tecnológicos na colheita florestal. Os avaliadores foram representados por três pessoas ligadas à empresa, as quais serviram como parâmetro no processo de avaliação ambiental. A seleção deles foi baseada no grau de experiência e conhecimento sobre a área técnica de colheita florestal. Eles utilizaram notas (escores) para avaliação dos componentes dos meios físico, biótico e antrópico. As notas atribuídas retrataram a variação do coeficiente de alteração, quando se comparou o método tradicional em relação ao inovado. Os coeficientes de alteração foram proporcionados, portanto, pela variação dos impactos em função do grau de comparação dos sistemas antes e após o processo de inovação. Esses coeficientes, multiplicados pelos seus fatores de ponderação (escala de ocorrência e fator de importância), resultaram nos coeficientes de impacto dos componentes dos respectivos indicadores dos meios físico, biótico e antrópico. Como foi considerou um processo de sucessão tecnológica, os sistemas compartilharam das mesmas realidades topográficas, edáficas e climáticas, entre outras, sendo consideradas, portanto, as mesmas condições de trabalho para o guincho arrastador e para o skidder de esteiras. No caso dos componentes do indicador atmosfera (gases de efeito estufa, material particulado, ruído e odores), estes foram analisados dentro da cabine, considerando, da mesma forma, os maquinários antes e após o processo de inovação. O processo de avaliação envolveu as etapas descritas a seguir.

\section{Definição dos indicadores e componentes avaliados}

A figura 1 ilustra os indicadores dos aspectos físico, biótico e antrópico, bem como seus respectivos componentes utilizados para avaliação de impacto ambiental da inovação tecnológica na colheita florestal.

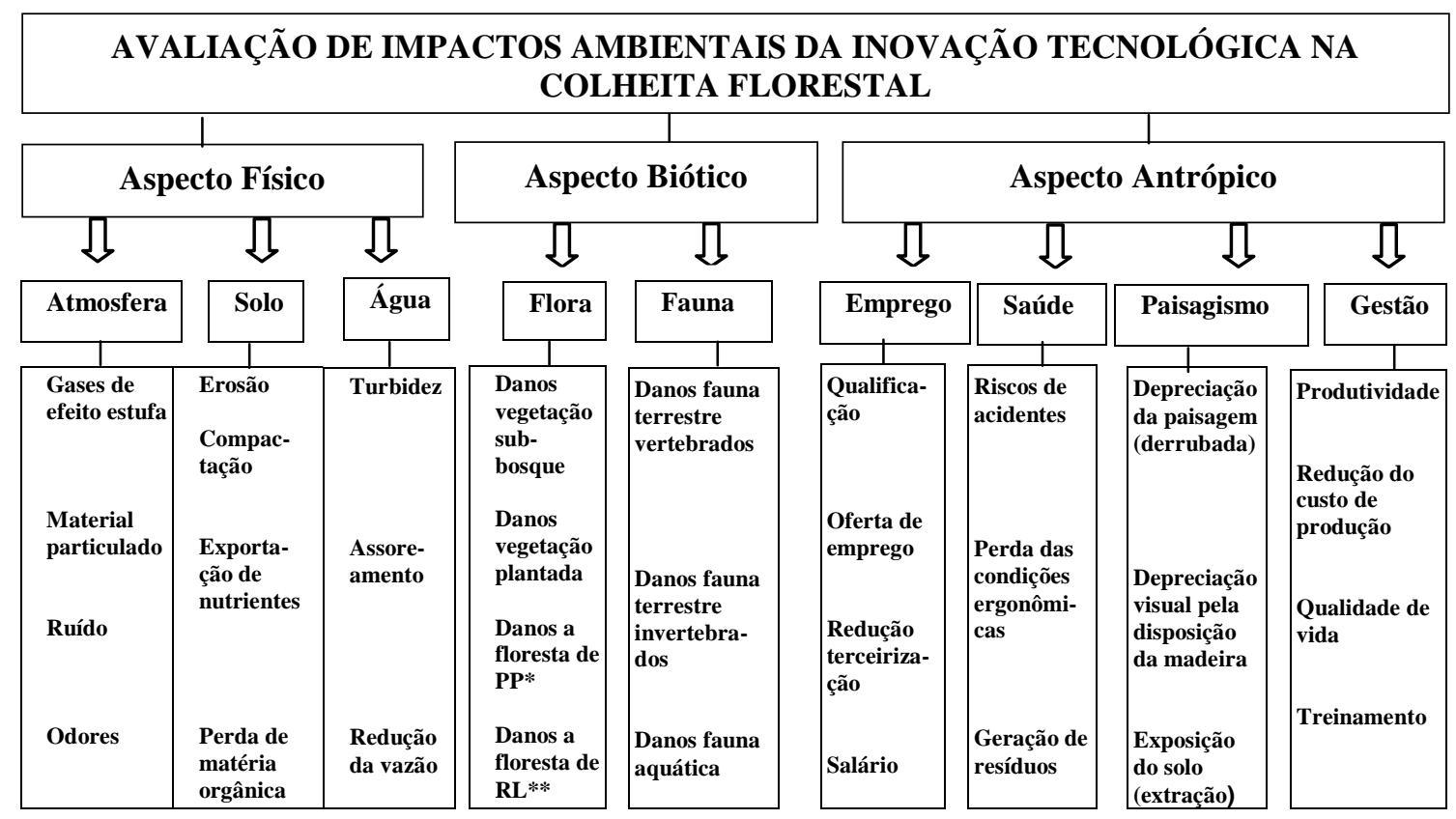

*: Preservação permanente; **: Reserva legal.

Figura 1. Diagrama utilizado para avaliação de impacto ambiental da inovação tecnológica na colheita florestal, apresentando os aspectos, indicadores e componentes - adaptado do sistema AMBITEC-AGRO (RODRIGUES et al., 2003).

Figure 1. Diagram used to evaluation of environmental impact of the technological innovation in the forest harvesting, presenting the aspects, indicators and components - adapted of the AMBITEC-AGRO system (RODRIGUES et al., 2003).

\section{Definição dos subsistemas de colheita florestal avaliados}

Foram avaliados os impactos ambientais no subsistema inovado (motosserra + skidder de esteiras), tomando como referência o modal de colheita tradicional (motosserra + guincho arrastador). 
Descrição dos subsistemas de colheita florestal avaliados

No subsistema inovado, a motosserra foi utilizada apenas na derrubada, sendo o desgalhamento e destopamento das árvores executados de forma manual, com auxílio de machadinhas. A extração foi realizada por meio de um skidder de esteiras da marca Caterpillar, modelo 527, responsável pelo arraste dos feixes de toras até as margens das estradas, onde eram processadas com auxílio da garra traçadora. No subsistema tradicional, as operações de corte (derrubada, desgalhamento, destopamento e traçamento), foram realizadas de forma semimecanizada, com motosserra, e as árvores abatidas foram traçadas num comprimento de 2,20 metros, com os toretes resultantes empilhados dentro do talhão e, a partir daí, arrastados por meio de um guincho acoplado a um trator agrícola Massey Ferguson, modelo 265.

\section{Elaboração das matrizes}

Elaboraram-se nove matrizes de ponderação, sendo três referentes ao aspecto físico, duas ao aspecto biótico e quatro ao aspecto antrópico. Cada indicador, com seus respectivos componentes (Figura 1), resultou numa matriz de ponderação, de forma a proceder à avaliação de impacto ambiental da inovação tecnológica na colheita florestal.

\section{Coleta de dados}

O primeiro passo para a obtenção dos dados foi a ponderação dos componentes de cada indicador, de acordo com a escala de ocorrência (Figura 1) e fator importância, conforme ilustrado abaixo.

Tabela 1. Escala de ocorrência para ponderação dos componentes avaliados.

Table1. Inclusion scale for consideration of the appraised components.

\begin{tabular}{lc}
\hline Escala de ocorrência & Fatores de ponderação \\
\hline Impacto pontual & 1 \\
Impacto local & 2 \\
Impacto no entorno & 5 \\
\hline Impacto pontual: impacto que se restringe à área de corte. Impacto local: impacto de maior \\
abrangência em relação ao impacto pontual, restringindo-se à área do projeto. Impacto no entorno: \\
impacto de maior abrangência em relação ao impacto local, extravasando a área do projeto.
\end{tabular}

a) Escala de ocorrência

Esses fatores de ponderação foram selecionados de forma a exprimir o aumento do impacto conforme a escala espacial, agravando-o quando a escala de ocorrência estende-se além dos limites da unidade produtiva (RODRIGUES et al., 2003).

b) Fator de importância

Consistiu numa etapa para normalização devido aos diferentes números de componentes de cada indicador e também para consideração do peso relativo de impacto nos respectivos componentes avaliados. O somatório do fator de importância de todos componentes de um determinado indicador resultou em +1 ou -1 (um). Neste trabalho, o fator de importância adotado foi aquele obtido da média das três avaliações, sendo estas realizadas por pessoas ligadas à área de colheita florestal da empresa, sendo um com formação técnica em meio ambiente, um com especialização em nível de pós-graduação em gestão ambiental e um com formação em engenharia florestal, com pós-graduação em ciência florestal. Cada avaliador expressou sua ponderação de acordo com o grau de importância dos respectivos componentes.

Após a ponderação, procedeu-se o preenchimento das matrizes, utilizando-se os coeficientes de alteração previamente estabelecidos (Tabela 2). Elas foram preenchidas de forma individual, tomando como referência técnicos da área de colheita florestal que vivenciaram o progresso tecnológico em estudo. A avaliação foi realizada com base na análise de três representantes. Cada um preencheu um total de 9 matrizes referentes aos indicadores definidos na figura 1.

\section{Determinação do coeficiente e do índice geral de impacto}

Os coeficientes de impacto foram determinados através da multiplicação dos coeficientes de alteração (Tabela 2) pelos fatores de ponderação (escala de ocorrência e fator de importância). Esses coeficientes foram calculados para todos os componentes de cada um dos indicadores, que no somatório resultou no coeficiente de impacto do respectivo indicador, enquadrado numa escala de -15 a +15 , 
captando assim as alterações positivas e negativas proporcionadas pela diversificação tecnológica no subsistema avaliado.

Tabela 2. Coeficientes de alteração padronizados para avaliação quantitativa de impactos ambientais da inovação tecnológica na colheita florestal.

Table 2. Alteration coefficients standardized for quantitative evaluation of environmental impacts of the technological innovation in the forest harvesting.

\begin{tabular}{lc}
\hline Efeito da tecnologia na atividade de colheita florestal & Coeficiente de alteração do componente \\
\hline Grande aumento no componente & +3 \\
Moderado aumento no componente & +1 \\
Componente inalterado & 0 \\
Moderada diminuição do componente & -1 \\
Grande diminuição do componente & -3 \\
\hline
\end{tabular}

Para uma análise global, os nove indicadores contemplados no estudo foram também ponderados (somatório igual a 1), sendo os valores resultantes dessa ponderação multiplicados pelos respectivos coeficientes de impactos dos indicadores, como forma de se determinar o índice geral de impacto no processo de inovação tecnológica analisado.

\section{RESULTADOS E DISCUSSÃO}

Avaliação de impactos ambientais da inovação tecnológica na colheita florestal

O processo de inovação em estudo proporcionou alterações ambientais em relação aos indicadores dos aspectos físico, biótico e antrópico, as quais se encontram descritas abaixo.

\section{Atmosfera}

A atmosfera foi avaliada mediante estudo dos seguintes componentes: gases de efeito estufa, material particulado, ruído e odores. Os gases de efeito estufa apresentaram alterações positiva e negativa, em nível moderado, quando da adoção do subsistema inovado, mantendo-se inalterados em uma das repetições (Figura 2). Com relação ao material particulado, a inovação promoveu moderada redução em duas avaliações (Figura 2). O arraste das pilhas de madeira com guincho (pré-inovação) proporcionou maior exposição do solo quando comparado ao skidder de esteiras, favorecendo consequentemente a emissão de maior quantidade de particulados para a atmosfera. Os componentes "ruído" e "odores" também apresentaram coeficiente de alteração negativo, decorrente do processo de inovação (Figura 2).

\begin{tabular}{|c|c|c|c|c|c|c|c|c|c|c|c|c|c|c|c|}
\hline \multirow{4}{*}{\begin{tabular}{|c} 
Atmosfera \\
Subsistemas \\
Motosserra + guincho arrastador \\
Motosserra + skidder de esteiras \\
\\
Fatores de \\
ponderação \\
Repeticões
\end{tabular}} & \multicolumn{12}{|c|}{$\begin{array}{l}\text { Indicador do aspecto físico } \\
\text { Componentes: }\end{array}$} & \multicolumn{3}{|c|}{$\begin{array}{l}\text { Averiguação dos } \\
\text { fatores de } \\
\text { ponderação }\end{array}$} \\
\hline & \multicolumn{3}{|c|}{$\begin{array}{l}\text { Gases de } \\
\text { efeito estufa }\end{array}$} & \multicolumn{3}{|c|}{$\begin{array}{l}\text { Material } \\
\text { particulado }\end{array}$} & \multicolumn{3}{|c|}{ Ruído } & \multicolumn{3}{|c|}{ Odores } & & & \\
\hline & \multicolumn{3}{|c|}{$-0,18$} & \multicolumn{3}{|c|}{$-0,17$} & \multicolumn{3}{|c|}{$-0,55$} & \multicolumn{3}{|c|}{$-0,10$} & \multicolumn{3}{|c|}{$-1,00$} \\
\hline & $\mathbf{R}_{1}$ & $\mathbf{R}_{2}$ & $\mathbf{R}_{3}$ & $\mathbf{R}_{\mathbf{1}}$ & $\mathbf{R}_{\mathbf{2}}$ & $\mathbf{R}_{\mathbf{3}}$ & $\mathbf{R}_{\mathbf{1}}$ & $\mathbf{R}_{2}$ & $\mathbf{R}_{\mathbf{3}}$ & $\mathbf{R}_{1}$ & $\mathbf{R}_{2}$ & $\mathbf{R}_{3}$ & & & \\
\hline Impacto Pontual & & - & - & -1 & $\mathbf{0}$ & -1 & - & - & - & $\mathbf{0}$ & $\mathbf{0}$ & -1 & & & \\
\hline Impacto Local & - & - & - & - & - & - & -1 & -1 & -1 & - & - & - & & & \\
\hline Impacto Entorno & +1 & $\mathbf{0}$ & -1 & - & - & - & - & - & - & - & - & - & & & \\
\hline $\begin{array}{l}\text { Coeficiente de impacto: } \\
\text { Coeficiente de impacto }(\end{array}$ & $\begin{array}{l}-0,90 \\
=C\end{array}$ & 0,00 & $\begin{array}{l}0,91 \\
\text { te d }\end{array}$ & $\begin{array}{l}0,17 \\
\text { Iter }\end{array}$ & a,00 & 0,17 & & $\begin{array}{c}1,10 \\
\text { nde }\end{array}$ & 1,10 & 0,00 & 0,00 & $\mathbf{0 , 1 0}$ & $\begin{array}{c}\text { R1 } \\
0.37\end{array}$ & $\begin{array}{l}\sum \mathrm{CI} \\
\mathbf{R 2} \\
1.10\end{array}$ & $\begin{array}{c}\text { R3 } \\
2.27\end{array}$ \\
\hline
\end{tabular}

Figura 2. Matriz de ponderação dos componentes de um indicador do aspecto físico (atmosfera), do sistema de avaliação de impacto ambiental da inovação tecnológica da colheita florestal.

Figure 2. Weighing matrices of the components of an indicator of the physical aspect (atmosphere), of the system of evaluation of environmental impact of the technological innovation in the forest harvesting. 
O coeficiente total de impacto mostrou-se positivo nas três repetições, refletindo, dessa forma, melhorias ambientais para o indicador atmosfera quando da adoção do processo de inovação.

\section{Solo}

Esse indicador foi composto pelos seguintes componentes: erosão, compactação, exportação de nutrientes e perda de matéria orgânica. A exposição do solo pelo arraste das pilhas de madeira no subsistema tradicional fez com que este apresentasse um adicional de impacto em relação à erosão, o que, consequentemente, refletiu na redução do respectivo componente quando da adoção do subsistema inovado (Figura 3).

A compactação do solo apresentou aumento moderado nas três avaliações quando da adoção do processo de inovação, sendo observada a mesma tendência, em duas repetições, para o componente "exportação de nutrientes" (Figura 3). No subsistema tradicional (motosserra + guincho arrastador), a madeira foi processada dentro dos talhões, favorecendo, portanto, a manutenção dos nutrientes no sítio. Após a inovação (motosserra + skidder de esteiras), a madeira passou a ser processada nas margens das estradas, o que potencializou as perdas de nutrientes. A perda de matéria orgânica do solo reduziu moderadamente na maioria das repetições quando da adoção do subsistema inovado (Figura 3). Isso significa dizer que o subsistema tradicional apresentou um adicional de impacto em relação a esse componente. A explicação nesse caso estaria relacionada à maior exposição do solo orgânico durante o arraste dos toretes com o guincho arrastador.

A erosão foi o componente de maior evidência nessa avaliação, o que ocorreu em função de sua ponderação significativa em relação ao fator de importância $(-0,43)$. O coeficiente total de impacto mostrou-se positivo em duas das três repetições (Figura 3), indicando que o subsistema tradicional foi mais impactante em relação ao subsistema inovado. Esse resultado está de acordo com os obtidos por Freitas (2004), o qual detectou, para a atividade de extração, valores mais expressivos de impacto quando o processo era realizado por meio do guincho arrastador.

\begin{tabular}{|c|c|c|c|c|c|c|c|c|c|c|c|c|c|c|c|}
\hline \multirow{2}{*}{\begin{tabular}{l}
\multicolumn{1}{c}{ Solo } \\
Subsistemas \\
Motosserra + guincho arrastador \\
Motosserra + skidder de esteiras \\
Fatores de \\
ponderação
\end{tabular}} & \multicolumn{12}{|c|}{$\begin{array}{l}\text { Indicador do aspecto físico } \\
\text { Componentes: }\end{array}$} & \multicolumn{3}{|c|}{$\begin{array}{l}\text { Averiguação dos } \\
\text { fatores de } \\
\text { ponderação }\end{array}$} \\
\hline & \multicolumn{3}{|c|}{ Erosão } & & $-0,27$ & & & $-0,14$ & & & $-0,16$ & & & $-1,0$ & \\
\hline Repeticões & $\mathbf{R}_{1}$ & $\mathbf{R}_{\mathbf{2}}$ & $\mathbf{R}_{3}$ & $\mathbf{R}_{\mathbf{1}}$ & $\mathbf{R}_{\mathbf{2}}$ & $\mathbf{R}_{\mathbf{3}}$ & $\mathbf{R}_{1}$ & $\mathbf{R}_{2}$ & $\mathbf{R}_{\mathbf{3}}$ & $\mathbf{R}_{1}$ & $\mathbf{R}_{2}$ & $\mathbf{R}_{\mathbf{3}}$ & & & \\
\hline Impacto Pontual & -1 & -1 & -1 & +1 & +1 & +1 & +1 & 0 & +1 & +1 & -1 & -1 & & & \\
\hline Impacto Local & - & - & - & - & - & - & - & - & - & - & - & - & & & \\
\hline Impacto Entorno & - & - & - & - & - & - & - & - & - & - & - & - & & & \\
\hline $\begin{array}{l}\text { Coeficiente de impacto: } \\
\text { Coeficiente de impacto }(\mathrm{Cl})\end{array}$ & $\begin{array}{l}2,15 \\
\text { Coef }\end{array}$ & $\begin{array}{r}2,15 \\
\text { ciente }\end{array}$ & $\begin{array}{l}2,15 \\
\text { le alt }\end{array}$ & $\begin{array}{l}-1,35 \\
\text { raçã }\end{array}$ & $\begin{array}{l}-1,35 \\
* \text { Fat }\end{array}$ & $\begin{array}{l}-1,35 \\
\text { ores }\end{array}$ & $\begin{array}{r}-0,70 \\
\text { de pon }\end{array}$ & $\begin{array}{r}0,00 \\
\text { eraç }\end{array}$ & $-0,70$ & $-0,80$ & 0,80 & $\mathbf{0 , 8 0}$ & $\begin{array}{l}\mathbf{R} 1 \\
\mathbf{- 0 , 7 0}\end{array}$ & $\begin{array}{l}\sum \mathrm{Cl} \\
\mathrm{R} 2 \\
\mathbf{1 , 6 0}\end{array}$ & $\begin{array}{l}\text { R3 } \\
\mathbf{0 , 9 0}\end{array}$ \\
\hline
\end{tabular}

Figura 3. Matriz de ponderação dos componentes de um indicador do aspecto físico (solo), do sistema de avaliação de impacto ambiental da inovação tecnológica da colheita florestal.

Figure 3. Weighing matrices of the components of an indicator of the physical aspect (soil), of the system of evaluation of environmental impact of the technological innovation in the forest harvesting.

\section{Água}

Esse indicador foi avaliado mediante os seguintes parâmetros: turbidez, assoreamento e vazão. A turbidez e o assoreamento apresentaram redução quando da adoção do subsistema inovado, mostrando, a extração com o guincho, um adicional de impacto em relação aos referidos componentes (Figura 4). Nessa operação, a exposição do solo se torna mais evidente quando comparada ao arraste com skidder de esteiras, favorecendo de maneira mais ativa a ocorrência de impactos indiretos sobre os corpos hídricos (FREITAS, 2008). O maior efeito da compactação no subsistema inovado fez com que este apresentasse um adicional de impacto em relação à vazão, sendo esse aspecto observado por dois dos três avaliadores (Figura 4). 
O componente que apresentou maior coeficiente de alteração foi o assoreamento, o que proporcionou inclusive coeficiente de impacto bastante expressivo em duas repetições (Figura 4). O coeficiente total de impacto mostrou-se positivo nas três avaliações, indicando que o processo de inovação em estudo contribuiu para melhoria dos aspectos ambientais relacionados ao indicador em questão.

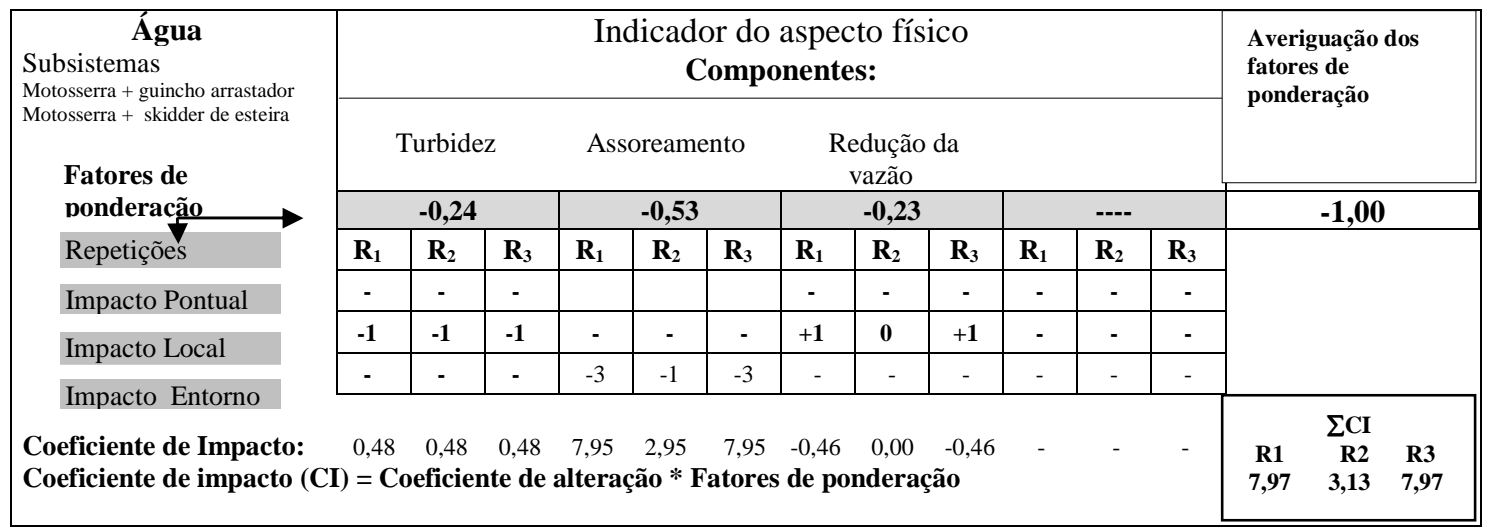

Figura 4. Matriz de ponderação dos componentes de um indicador do aspecto físico (água), do sistema de avaliação de impacto ambiental da inovação tecnológica da colheita florestal.

Figure 4. Weighing matrices of the components of an indicator of the physical aspect (water), of the system of evaluation of environmental impact of the technological innovation in the forest harvesting.

Flora

A flora foi analisada com base nos danos ocorridos na vegetação do sub-bosque, na vegetação plantada e também na floresta do entorno. Os danos inerentes à vegetação presente nos talhões (sub-bosque e plantada) e no entorno (floresta de reserva legal e de preservação permanente) apresentaram alterações positivas em duas repetições, quando da adoção do subsistema inovado (Figura 5). A inovação tecnológica proporcionou, de forma global, aumento dos danos ambientais em relação à vegetação avaliada, o que pode ser constatado pelos coeficientes negativos de impacto (Figura 5). Embora esses danos tenham sido agravados no subsistema inovado, Machado et al. (1990) relataram problemas ambientais relacionados à vegetação plantada (cepas) quando da extração de madeira pelo guincho arrastador em plantio de eucalipto.

\begin{tabular}{|c|c|c|c|c|c|c|c|c|c|c|c|c|c|c|}
\hline \multirow{4}{*}{\begin{tabular}{l}
\multicolumn{1}{c}{ Flora } \\
Subsistemas \\
Motosserra + guincho arrastador \\
Motosserra + skidder de esteiras \\
Fatores de \\
ponderação \\
Repeticões
\end{tabular}} & \multicolumn{12}{|c|}{$\begin{array}{l}\text { Indicador do aspecto biótico } \\
\text { Componentes: }\end{array}$} & \multirow{2}{*}{\multicolumn{2}{|c|}{$\begin{array}{l}\text { Averiguação dos } \\
\text { fatores de } \\
\text { ponderação }\end{array}$}} \\
\hline & \multicolumn{3}{|c|}{$\begin{array}{l}\text { Danos vegetação } \\
\text { sub-bosque }\end{array}$} & \multicolumn{3}{|c|}{$\begin{array}{l}\text { Danos vegetação } \\
\text { plantada }\end{array}$} & \multicolumn{3}{|c|}{$\begin{array}{c}\text { Danos a floresta } \\
\text { preservação perm. }\end{array}$} & \multicolumn{3}{|c|}{$\begin{array}{l}\text { Danos a floresta } \\
\text { reserva legal }\end{array}$} & & \\
\hline & \multicolumn{3}{|c|}{$-0,15$} & \multicolumn{3}{|c|}{$-0,19$} & \multicolumn{3}{|c|}{$-0,36$} & \multicolumn{3}{|c|}{$-0,30$} & \multicolumn{2}{|r|}{$-1,00$} \\
\hline & $\mathbf{R}_{\mathbf{1}}$ & $\mathbf{R}_{2}$ & $\mathbf{R}_{\mathbf{3}}$ & $\mathbf{R}_{1}$ & $\mathbf{R}_{2}$ & $\mathbf{R}_{\mathbf{3}}$ & $\mathbf{R}_{1}$ & $\mathbf{R}_{2}$ & $\mathbf{R}_{\mathbf{3}}$ & $\mathbf{R}_{\mathbf{1}}$ & $\mathbf{R}_{2}$ & $\mathbf{R}_{\mathbf{3}}$ & & \\
\hline Impacto Pontual & 3 & $\mathbf{0}$ & 1 & 3 & $\mathbf{0}$ & 1 & & & & & & & & \\
\hline Impacto Local & - & - & - & - & - & - & 1 & $\mathbf{0}$ & 1 & 1 & 0 & -1 & & \\
\hline Impacto Entorno & - & - & - & - & - & - & - & - & - & - & - & - & & \\
\hline $\begin{array}{l}\text { Coeficiente de impacto: } \\
\text { Coeficiente de impacto }\end{array}$ & $\begin{array}{l}-0,45 \\
I)=(\end{array}$ & 0,00 & $\begin{aligned}-0,15 \\
\text { ite d }\end{aligned}$ & $\begin{array}{l}-\mathbf{0 , 5 7} \\
\text { alter }\end{array}$ & $\begin{array}{l}\mathbf{0 , 0 0} \\
\text { ão * }\end{array}$ & $-0,1$ & $-0,7$ & 0,0 & $\sim-0,72$ & $-0,60$ & 0,00 & $-0,60$ & $\begin{array}{c}\mathrm{R} 1 \\
-2,34\end{array}$ & $\begin{array}{cc}\Sigma \mathrm{CI} & \\
\mathrm{R} 2 & \mathrm{R3} \\
0,00 & -1,66\end{array}$ \\
\hline
\end{tabular}

Figura 5. Matriz de ponderação dos componentes de um indicador do aspecto biótico (flora), do sistema de avaliação de impacto ambiental da inovação tecnológica da colheita florestal.

Figure 5. Weighing matrices of the components of an indicator of the biotic aspect (plants), of the system of evaluation of environmental impact of the technological innovation in the forest harvesting. 


\section{Fauna}

Este indicador foi analisado com base nos danos inerentes a fauna terrestre (vertebrados e invertebrados), bem como aqueles relacionados à fauna aquática. Todos componentes apresentaram alterações positivas, em nível moderado, quando da adoção do subsistema inovado (Figura 6). Em uma das repetições $\left(R_{2}\right)$, os componentes mantiveram-se inalterados, resultando coeficiente total de impacto igual a zero para o respectivo indicador. A substituição do subsistema tradicional (motosserra + guincho arrastador) pelo inovado (motosserra + skidder de esteiras) proporcionou, na maioria das repetições, coeficiente total de impacto negativo, mostrando portanto que a referida mudança não trouxe melhorias ambientais em relação ao indicador em questão.

\begin{tabular}{|c|c|c|c|c|c|c|c|c|c|c|c|c|c|c|}
\hline \multirow{4}{*}{\begin{tabular}{l}
\multicolumn{1}{c}{ Fauna } \\
Subsistemas \\
Motosserra + guincho arrastador \\
Motosserra + skidder de esteiras \\
Fatores de \\
ponderação \\
Repeticões
\end{tabular}} & \multicolumn{12}{|c|}{$\begin{array}{l}\text { Indicador do aspecto biótico } \\
\text { Componentes: }\end{array}$} & \multirow{2}{*}{\multicolumn{2}{|c|}{$\begin{array}{l}\text { Averiguação dos } \\
\text { fatores de } \\
\text { ponderação }\end{array}$}} \\
\hline & \multicolumn{3}{|c|}{$\begin{array}{l}\text { Danos à fauna } \\
\text { ter. (vertebrados) }\end{array}$} & \multicolumn{3}{|c|}{$\begin{array}{l}\text { Danos à fauna } \\
\text { ter. (invertebrados) }\end{array}$} & \multicolumn{3}{|c|}{$\begin{array}{l}\text { Danos à fauna } \\
\text { aquática }\end{array}$} & & & & & \\
\hline & \multicolumn{3}{|c|}{$-0,32$} & \multicolumn{3}{|c|}{$-0,40$} & \multicolumn{3}{|c|}{$-0,28$} & \multicolumn{3}{|c|}{$\begin{array}{ll}--- \\
--\end{array}$} & & $-1,00$ \\
\hline & $\mathbf{R}_{1}$ & $\mathbf{R}_{2}$ & $\mathbf{R}_{3}$ & $\mathbf{R}_{1}$ & $\mathbf{R}_{2}$ & $\mathbf{R}_{\mathbf{3}}$ & $\mathbf{R}_{1}$ & $\mathbf{R}_{2}$ & $\mathbf{R}_{\mathbf{3}}$ & $\mathbf{R}_{1}$ & $\mathbf{R}_{2}$ & $\mathbf{R}_{3}$ & & \\
\hline Impacto Pontual & 1 & $\mathbf{0}$ & 1 & 1 & $\mathbf{0}$ & 1 & - & - & - & - & - & - & & \\
\hline Impacto Local & - & - & - & - & - & - & 1 & 0 & 1 & - & - & - & & \\
\hline Impacto Entorno & - & - & - & - & - & - & - & - & - & - & - & - & & \\
\hline $\begin{array}{l}\text { Coeficiente de impacto: } \\
\text { Coeficiente de impacto }\end{array}$ & $\begin{array}{l}-0,32 \\
I)=\end{array}$ & & 0,32 & & & & & 0,00 & $-0,56$ & - & - & - & $\begin{array}{c}R 1 \\
-1,28\end{array}$ & $\begin{array}{rr}R 2 & R 3 \\
0,00 & -1,28\end{array}$ \\
\hline
\end{tabular}

Figura 6. Matriz de ponderação dos componentes de um indicador do aspecto biótico (fauna), do sistema de avaliação de impacto ambiental da inovação tecnológica da colheita florestal.

Figure 6. Weighing matrices of the components of an indicator of the biotic aspect (fauna), of the system of evaluation of environmental impact of the technological innovation in the forest harvesting.

\section{Saúde}

O indicador saúde foi analisado em relação aos riscos de acidentes, perda das condições ergonômicas e geração de resíduos. Os riscos de acidentes apresentaram redução em duas repetições quando da adoção do subsistema inovado (Figura 7). A redução do número de acidentes esteve relacionada com a diminuição do número de operadores na extração (substituição do guincho arrastador pelo skidder de esteiras). Deve-se ainda levar em consideração os problemas inerentes à extração com o guincho arrastador, já que nesse caso os operadores têm que descer relevos acidentados para fazer o engate dos cabos às pilhas de madeira, aspecto este que potencializa os riscos de acidentes. Com o processo de inovação, as operações passaram também a ser realizadas de forma menos dispendiosa, ficando os operadores livres dos gases e poeiras, uma vez que o skidder de esteiras apresenta cabines fechadas, diferentemente do que é observado no trator agrícola utilizado como guincho arrastador. Esse novo cenário proporcionou, portanto, avanços nos aspectos ergonômicos (Figura 2). Cabe ressaltar que a inovação condicionou a ocorrência de outros problemas ergonômicos. Souza et al. (2003) identificaram na operação de extração com o skidder de esteiras operadores expostos a trabalho com grande quantidade de repetições de movimentos de alto grau de rotina, às vezes sob esforços e postura inadequadas, com possíveis consequências para a saúde.

O saldo total de impacto mostrou-se positivo na maioria das repetições, retratando, no contexto geral, melhorias para o respectivo indicador quando da adoção do subsistema inovado.

\section{Emprego}

O indicador emprego foi composto pelos seguintes componentes: qualificação, oferta de empregos, redução da terceirização e salário. A qualificação e o salário mostraram alterações positivas em todas as repetições (Figura 8). A inovação tecnológica no processo de arraste (guincho arrastador para skidder de esteiras) fez com que a empresa buscasse operadores com maior nível de instrução para 
realização da referida operação, o que possibilitou melhorias não apenas na qualificação profissional, mas também em relação à remuneração.

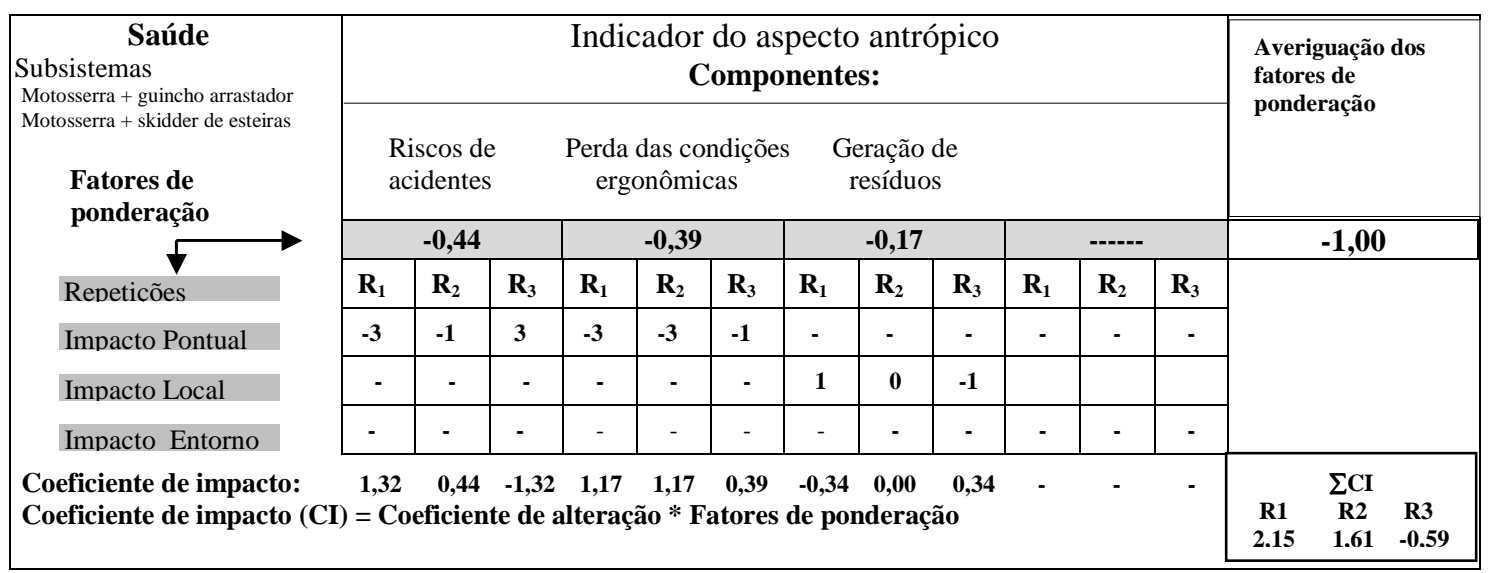

Figura 7. Matriz de ponderação dos componentes de um indicador do aspecto antrópico (saúde), do sistema de avaliação de impacto ambiental da inovação tecnológica da colheita florestal.

Figure 7. Weighing matrices of the components of an indicator of the antropic aspect (health), of the system of evaluation of environmental impact of the technological innovation in the forest harvesting.

A oferta de empregos apresentou moderada retração em todas as avaliações (Figura 8). Isso ocorreu pelo fato de o skidder de esteiras exigir menor contingente de mão de obra na operação de arraste em relação ao guincho arrastador.

O coeficiente total de impacto apresentou valores positivos nas três repetições, mostrando, portanto, que o processo de inovação trouxe benefícios de cunho social relacionados ao indicador em questão.

\begin{tabular}{|c|c|c|c|c|c|c|c|c|c|c|c|c|c|c|c|}
\hline \multirow{3}{*}{\begin{tabular}{|c} 
Emprego \\
Subsistemas \\
Motosserra + guincho arrastador \\
Motosserra + skidder de esteiras \\
\\
Fatores de \\
ponderação \\
\end{tabular}} & \multicolumn{12}{|c|}{$\begin{array}{l}\text { Indicador do aspecto antrópico } \\
\text { Componentes: }\end{array}$} & \multicolumn{3}{|c|}{$\begin{array}{l}\text { Averiguação dos } \\
\text { fatores de } \\
\text { ponderação }\end{array}$} \\
\hline & \multirow{2}{*}{\multicolumn{3}{|c|}{ Qualificação }} & \multicolumn{3}{|c|}{$\begin{array}{l}\text { Oferta de } \\
\text { emprego }\end{array}$} & \multicolumn{3}{|c|}{$\begin{array}{l}\text { Redução da } \\
\text { terceirização }\end{array}$} & \multicolumn{3}{|c|}{ Salário } & & & \\
\hline & & & & \multicolumn{3}{|c|}{0,20} & \multicolumn{3}{|c|}{0,23} & \multicolumn{3}{|c|}{0,27} & \multicolumn{3}{|c|}{$-1,00$} \\
\hline Repeticões & $\mathbf{R}_{1}$ & $\mathbf{R}_{2}$ & $\mathbf{R}_{\mathbf{3}}$ & $\mathbf{R}_{1}$ & $\mathbf{R}_{2}$ & $\mathbf{R}_{3}$ & $\mathbf{R}_{1}$ & $\mathbf{R}_{\mathbf{2}}$ & $\mathbf{R}_{3}$ & $\mathbf{R}_{1}$ & $\mathbf{R}_{2}$ & $\mathbf{R}_{3}$ & & & \\
\hline Impacto Pontual & 3 & 1 & 1 & - & - & - & - & - & - & 1 & 1 & 1 & & & \\
\hline Impacto Local & - & - & - & - & - & - & - & - & - & - & - & - & & & \\
\hline Impacto Entorno & - & - & - & -1 & -1 & -1 & 1 & 1 & 1 & - & - & - & & & \\
\hline \multicolumn{13}{|c|}{ 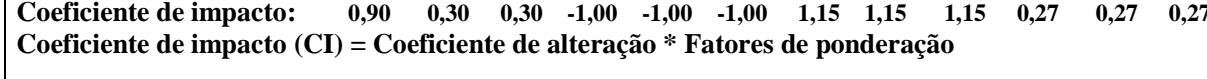 } & $\begin{array}{c}\text { R1 } \\
1.32\end{array}$ & $\begin{array}{c}\sum \mathbf{C I} \\
\mathbf{R 2} \\
\mathbf{0 . 7 2}\end{array}$ & $\begin{array}{l}\text { R3 } \\
0.72\end{array}$ \\
\hline
\end{tabular}

Figura 8. Matriz de ponderação dos componentes de um indicador do aspecto antrópico (emprego), do sistema de avaliação de impacto ambiental da inovação tecnológica da colheita florestal.

Figure 8. Weighing matrices of the components of an indicator of the antropic aspect (employment), of the system of evaluation of environmental impact of the technological innovation in the forest harvesting.

\section{Paisagismo}

O paisagismo foi avaliado mediante estudo dos seguintes componentes: depreciação da paisagem pela remoção da floresta, depreciação visual pela disposição da madeira no talhão e exposição do solo no

FLORESTA, Curitiba, PR, v. 41, n. 3, p. 519-532, jul./set. 2011.

Freitas, L. C. de et al. 
processo de extração. A depreciação da paisagem pela remoção da floresta apresentou redução em duas repetições quando da adoção do subsistema inovado (Figura 9). No subsistema tradicional, a madeira é guinchada para as partes mais altas do terreno, favorecendo a depreciação do aspecto paisagístico. No caso da extração com o skidder de esteiras, a madeira pode ser arrastada para as áreas de baixada, proporcionando, nessas circunstâncias, atenuação dos impactos visuais. A depreciação visual causada pela disposição da madeira no talhão apresentou grande redução em uma das repetições quando da adoção do subsistema inovado (Figura 9). O feixe de toras compridas deixadas nos talhões, para extração com o skidder de esteiras, acabou proporcionando uma melhor qualidade visual quando comparada aos feixes de toretes deixados nas áreas de corte para extração com o guincho arrastador.

\begin{tabular}{|c|c|c|c|c|c|c|c|c|c|c|c|c|c|c|c|}
\hline \multirow{4}{*}{\begin{tabular}{|l}
\multicolumn{2}{|c}{ Paisagismo } \\
Subsistemas \\
Motosserra + guincho arrastador \\
Motosserra + skidder de esteiras \\
\\
Fatores de \\
ponderação
\end{tabular}} & \multicolumn{12}{|c|}{$\begin{array}{l}\text { Indicador do aspecto antrópico } \\
\text { Componentes: }\end{array}$} & \multicolumn{3}{|c|}{$\begin{array}{l}\text { Averiguação dos } \\
\text { fatores de } \\
\text { ponderacão }\end{array}$} \\
\hline & \multirow{2}{*}{\multicolumn{3}{|c|}{$\begin{array}{l}\text { Depreciação da } \\
\text { paisagem pela } \\
\text { remoção da flor. } \\
\mathbf{- 0 , 2 8}\end{array}$}} & \multirow{2}{*}{\multicolumn{3}{|c|}{$\begin{array}{l}\text { Depreciação visual } \\
\text { pela disp.da } \\
\text { madeira no talhão } \\
\mathbf{- 0 , 3 0}\end{array}$}} & \multicolumn{6}{|c|}{$\begin{array}{l}\text { Exposição do } \\
\text { solo no processo } \\
\text { de extração }\end{array}$} & & & \\
\hline & & & & & & & & $-0,42$ & & & ----- & & & $-1,00$ & \\
\hline & $\mathbf{R}_{1}$ & $\mathbf{R}_{2}$ & $\mathbf{R}_{3}$ & $\mathbf{R}_{1}$ & $\mathbf{R}_{2}$ & $\mathbf{R}_{\mathbf{3}}$ & $\mathbf{R}_{1}$ & $\mathbf{R}_{2}$ & $\mathbf{R}_{3}$ & $\mathbf{R}_{1}$ & $\mathbf{R}_{2}$ & $\mathbf{R}_{3}$ & & & \\
\hline Imnacto Pontual & - & - & - & - & - & - & - & - & - & - & - & - & & & \\
\hline 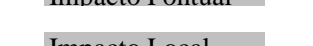 & - & - & - & 0 & -3 & 0 & -3 & 3 & 0 & - & - & - & & & \\
\hline Impacto Local & -1 & -3 & o & - & - & - & - & - & - & - & - & - & & & \\
\hline $\begin{array}{l}\text { Coeficiente de impacto: } \\
\text { Coeficiente de impacto (C) }\end{array}$ & & $\begin{array}{l}4,20 \\
\text { ficie }\end{array}$ & & & & & & $\begin{array}{l}-2,52 \\
\text { ddera }\end{array}$ & 0,00 & - & - & - & $\begin{array}{c}\text { R1 } \\
3,92\end{array}$ & $\begin{array}{c}\sum \mathrm{CI} \\
\mathbf{R 2} \\
\mathbf{3 , 4 8}\end{array}$ & $\begin{array}{c}\mathbf{R 3} \\
\mathbf{0 , 0 0}\end{array}$ \\
\hline
\end{tabular}

Figura 9. Matriz de ponderação dos componentes de um indicador do aspecto antrópico (paisagismo), do sistema de avaliação de impacto ambiental da inovação tecnológica da colheita florestal.

Figure 9. Weighing matrices of the components of an indicator of the antropic aspect (landscaping), of the system of evaluation of environmental impact of the technological innovation in the forest harvesting.

A exposição do solo pelo processo de extração mostrou alteração positiva e negativa quando da adoção do subsistema inovado. Por um lado, têm-se os fortes danos ao solo pelo arraste dos feixes de toretes (guinchamento), e, por outro, os impactos relacionados ao tráfego de máquinas e contato dos feixes de toras com o solo durante a extração com o skidder de esteiras.

O saldo total de impacto mostrou-se positivo em duas avaliações, sendo observada grande alteração do coeficiente de alteração em todos os componentes em uma das repetições (R2), conforme ilustrado na figura 9. Analisando no contexto geral, pode-se dizer que o processo de inovação contribuiu para melhoria dos aspectos paisagísticos.

\section{Gestão}

Esse indicador foi avaliado com base nos seguintes componentes: produtividade, redução do custo de produção, qualidade de vida e treinamento.

A produtividade apresentou aumento moderado quando da adoção do subsistema inovado (Figura 10). A redução da mão de obra, devido ao processo de inovação, proporcionou também a redução do custo de produção (Figura 10). Esse aspecto é de fundamental importância na colheita florestal, já que essa atividade, juntamente com o transporte, responde por cerca de 50\%, ou mais, dos custos totais da madeira posta na indústria (BAGIO; STOHR, 1978; REZENDE et al., 1983; MACHADO et al., 2008). A qualidade de vida e o treinamento nas operações de colheita apresentaram um aspecto positivo em virtude do processo de inovação (Figura 10). A melhoria dos salários pela qualificação profissional, bem como das condições ergonômicas pela modernização dos maquinários, refletiram de forma positiva na qualidade de vida dos trabalhadores. 


\section{Avaliação geral dos impactos ambientais da inovação tecnológica}

De acordo com a média das três repetições (Figura 11), sete indicadores apresentaram coeficiente de impacto positivo (atmosfera, água, solo, emprego, saúde, paisagismo e gestão) e dois mostraram coeficiente negativo (flora e fauna).

\begin{tabular}{|c|c|c|c|c|c|c|c|c|c|c|c|c|c|c|c|}
\hline \multirow{3}{*}{\begin{tabular}{|c} 
Gestão \\
Subsistemas \\
Motosserra + guincho arrastador \\
Motosserra + skidder de esteiras \\
\\
Fatores de \\
ponderação \\
\end{tabular}} & \multicolumn{12}{|c|}{$\begin{array}{l}\text { Indicador do aspecto antrópico } \\
\text { Componentes: }\end{array}$} & \multirow{2}{*}{\multicolumn{3}{|c|}{$\begin{array}{l}\text { Averiguação dos } \\
\text { fatores de } \\
\text { ponderação }\end{array}$}} \\
\hline & \multicolumn{3}{|c|}{ Produtividade } & \multicolumn{3}{|c|}{$\begin{array}{l}\text { Redução do } \\
\text { custo de produção }\end{array}$} & \multicolumn{3}{|c|}{$\begin{array}{l}\text { Qualidade } \\
\text { de vida }\end{array}$} & \multicolumn{3}{|c|}{ Treinamento } & & & \\
\hline & \multicolumn{3}{|c|}{0,25} & \multicolumn{3}{|c|}{0,35} & \multicolumn{3}{|c|}{$\mathbf{0 , 2 0}$} & \multicolumn{3}{|c|}{0,20} & & $-1,0$ & \\
\hline Repeticões & $\mathbf{R}_{\mathbf{1}}$ & $\mathbf{R}_{\mathbf{2}}$ & $\mathbf{R}_{\mathbf{3}}$ & $\mathbf{R}_{\mathbf{1}}$ & $\mathbf{R}_{\mathbf{2}}$ & $\mathbf{R}_{\mathbf{3}}$ & $\mathbf{R}_{\mathbf{1}}$ & $\mathbf{R}_{2}$ & $\mathbf{R}_{\mathbf{3}}$ & $\mathbf{R}_{\mathbf{1}}$ & $\mathbf{R}_{2}$ & $\mathbf{R}_{\mathbf{3}}$ & & & \\
\hline Impacto Pontual & 1 & $\mathbf{0}$ & 1 & -1 & -1 & $\mathbf{0}$ & 1 & 1 & 1 & 1 & 3 & 1 & & & \\
\hline Impacto Local & - & - & - & - & - & - & - & - & - & - & - & - & & & \\
\hline Impacto Entorno & - & - & - & - & - & - & - & - & - & - & - & - & & & \\
\hline $\begin{array}{l}\text { Coeficiente de impacto: } \\
\text { Coeficiente de impacto }(\mathrm{C}\end{array}$ & $\begin{array}{l}1,25 \\
=\mathrm{C}\end{array}$ & $\begin{array}{l}0,00 \\
\text { ficie }\end{array}$ & $\begin{array}{l}1,25 \\
\text { e de }\end{array}$ & $\begin{array}{r}-1,75 \\
\text { altera }\end{array}$ & $\begin{array}{l}-1,75 \\
10 * \mathbf{F}\end{array}$ & $\begin{array}{l}0,00 \\
\text { tore }\end{array}$ & & $\begin{array}{l}\text { 1,00 } \\
\text { der: }\end{array}$ & 1,00 & 1,00 & 3,00 & 1,00 & $\begin{array}{r}\mathbf{R} 1 \\
\mathbf{1 , 5 0}\end{array}$ & $\begin{array}{c}\sum \mathrm{C} \\
\mathrm{R2} \\
2,25\end{array}$ & $\begin{array}{c}\mathrm{R3} \\
\mathbf{3 , 2 5}\end{array}$ \\
\hline
\end{tabular}

Figura 10. Matriz de ponderação dos componentes de um indicador do aspecto antrópico (gestão), do sistema de avaliação de impacto ambiental da inovação tecnológica da colheita florestal.

Figure 10. Weighing matrices of the components of an indicator of the antropic aspect (management), of the system of evaluation of environmental impact of the technological innovation in the forest harvesting.
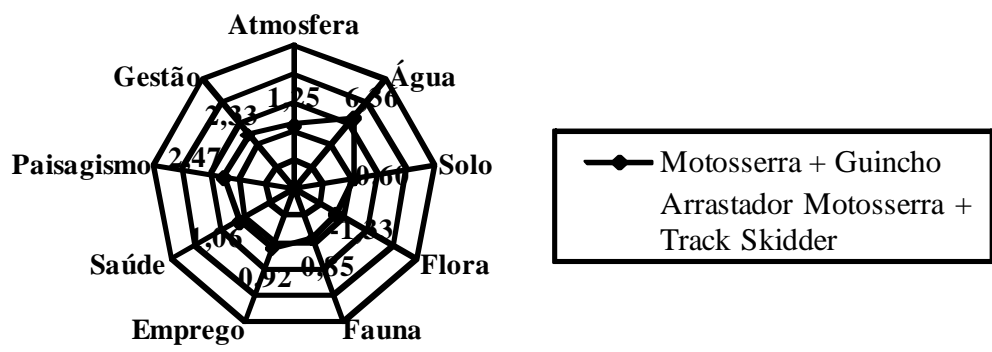

Figura 11. Coeficiente médio de impacto ambiental da inovação tecnológica da colheita florestal, nos aspectos físico, biótico e antrópico.

Figure 11. Medium coefficient of environmental impact of the technological innovation of the forest harvesting, in the aspects physicist, biotic and antropic.

Os resultados da tabela 3 ilustram o índice geral de impacto ambiental da inovação tecnológica na colheita florestal para o caso em estudo.

Embora o índice geral médio de impacto tenha atingido valor pouco expressivo em relação à escala, o resultado mostrou-se positivo, indicando assim melhorias ambientais quando da adoção do sistema inovado. 
Tabela 3. Ponderação dos indicadores e expressão do índice geral de impacto ambiental (médio) da inovação tecnológica na colheita florestal motosserra + guincho arrastador (antes da inovação), motosserra + skidder de esteiras (após inovação).

Table 3. Weighing of the indicators and expression of the general index of environmental impact (medium) of the technological innovation in the forest harvesting - chainsaw + cable skidder (before innovation), chainsaw + track skidder (after innovation).

\begin{tabular}{lccc}
\hline $\begin{array}{l}\text { Indicador de impacto } \\
\text { ambiental }\end{array}$ & $\begin{array}{c}\text { Peso do } \\
\text { indicador } \\
\text { (a) }\end{array}$ & $\begin{array}{c}\text { Coeficiente de impacto } \\
\text { ambiental médio } \\
(\mathbf{b})\end{array}$ & $\begin{array}{c}\text { Índice geral de impacto } \\
\text { médio } \\
\text { (a x b) }\end{array}$ \\
\hline Atmosfera & 0,05 & 1,25 & 0,06 \\
Solo & 0,13 & 0,60 & 0,08 \\
Água & 0,14 & 6,36 & 0,89 \\
Flora & 0,12 & $-1,33$ & $-0,16$ \\
Fauna & 0,12 & $-0,85$ & $-0,10$ \\
Emprego & 0,13 & 0,92 & 0,12 \\
Saúde & 0,14 & 1,06 & 0,15 \\
Paisagismo & 0,05 & 2,47 & 0,12 \\
Gestão & 0,12 & 2,33 & 0,28 \\
Averiguação da & Índice geral médio de impacto ambiental da inovação & 1,44 \\
ponderação 1 & tecnológica na colheita florestal: $1,44 *$ & \\
\hline (a): valores obtidos em função do grau de importância dos indicadores. *: Valor compreendido numa escala de -15 a +115.
\end{tabular}

\section{CONCLUSÕES}

- A substituição do guincho arrastador pelo skidder de esteiras, no processo de arraste, proporcionou, em termos médios, melhorias ambientais.

- Dada a abrangência em relação aos parâmetros avaliados, conclui-se que o melhor sistema (tradicional ou inovado) pode variar em função dos aspectos em análise, sejam estes pertencentes ao meio físico, biótico ou antrópico.

- A identificação e quantificação dos impactos nos sistema de colheita em questão constituíram um fator de grande importância no sentido de se adotar um controle ambiental para os impactos mais expressivos, através de medidas mitigadoras e potencializadoras.

- A variação restrita dos coeficientes de alteração contribuiu de forma decisiva para a diminuição do grau de subjetividade dos coeficientes de impacto.

- O perfil impactante do processo de inovação em questão se concretizou como um estudo de caso, tornando possível um comportamento diferenciado dos referidos impactos para outras realidades, tanto pelo nível de experiência dos avaliadores e grau de seu conhecimento sobre a temática como pelas condições topográficas diferenciadas ou mesmo pelo próprio grau de subjetividade implícito no método de avaliação.

\section{REFERÊNCIAS}

BAGIO, A. J.; STOHR, G. W. D. Resultados preliminares de um levantamento dos sistemas de exploração usados em florestas implantadas no Sul do Brasil. Floresta, Curitiba, v. 9, n. 2, p. 76 - 96, 1978.

BISSET, R. Methods for environmental impact assessment: a selective survey with case studies. In: BISWAS, A. K.; GEPING, Q. (Ed.). Environmental impact assessment for developing countries. London: Tycoly International, 1987. p. 3 - 64.

CANTER, L. W. Environmental impact assessment. New York: McGraw-Hill, 1996. 660 p.

EQUIPE TÉCNICA DURATEX. Colheita da madeira em florestas com baixo volume por árvore. In: SIMPÓSIO BRASILEIRO SOBRE COLHEITA E TRANSPORTE FLORESTAL, 4., 1999, Campinas. Anais... Viçosa: SIF/UFV, 1999. p. 54 - 72.

FREITAS, L. C. Avaliação quantitativa de impactos ambientais da colheita florestal em plantios equiâneos de eucalipto. 101 f. Dissertação (Mestrado em Ciência Florestal) - Universidade Federal de Viçosa, Viçosa, 2004. 
Avaliação de impactos ambientais da inovação tecnológica na colheita florestal. 118 p. Tese (Doutorado em Ciência Florestal) - Universidade Federal de Viçosa, Viçosa, 2008.

MACHADO, C. C.; IGNÁCIO, S. A.; VALE, A. B.; SOUZA JR., H. S. Efeito da extração de madeira com guincho arrastador na brotação do Eucalyptus alba. Revista Árvore, v. 14, n. 1, p. 55 - 60, 1990.

MACHADO, C. C.; SILVA, E. N.; PEREIRA, R. S. O setor florestal brasileiro e a colheita florestal. In: Colheita Florestal. Viçosa: UFV, 2008. p. 15 - 42.

MALINOVSKI, J. R.; CAMARGO, C. M. S.; MALINOVSKI, R. A.; MALINOVSKI, R. A. Sistemas. In: Colheita Florestal. Viçosa: UFV, 2008. p. 161-184.

REZENDE, J. L. P.; PEREIRA, A. R.; OLIVEIRA, A. D. Espaçamento ótimo para a produção de madeira. Revista Árvore, Viçosa, v. 7, n. 1, p. 30 - 43, 1983.

RODRIGUES, G. S.; CAMPANHOLA, C.; KITAMURA, P. C. Avaliação de impacto ambiental da inovação tecnológica agropecuária. Jaguariúna: Ambitec-Agro; Embrapa Meio Ambiente, 2003. 95 p. (Documento 34).

SEIXAS, F.; OLIVEIRA JÚNIOR, E. D. Compactação do solo devido ao tráfego de máquinas de colheita de madeira. Scientia Forestalis, n. 60, p. 73 - 87, 2001.

SOUZA, A. P.; MINETTI, L. J.; SILVA, K. R. Desafios da ergonomia na colheita florestal. In: SIMPÓSIO BRASILEIRO SOBRE COLHEITA E TRANSPORTE FLORESTAL, 6., 2003, Belo Horizonte. Anais... Viçosa: SIF/UFV, 2003. p. 217 - 232. 
